\title{
Research on Electrical and Electronic Laboratory Management System of Application-oriented University
}

\author{
Lirong Shen \\ Shanghai University of Medicine and Health Sciences, Shanghai, 201318, China
}

email: 360035344@qq.com

\begin{abstract}
Keywords: Laboratory Information Management System (LIMS); Function Module; Overall Lifecycle Management
\end{abstract}

\begin{abstract}
The traditional management methods of electrical and electronic laboratory have not satisfied the development of application-oriented university. To educate student comprehensive quality and creation ability, improve the quality of experiment teaching and increase the working efficiency for lab managers, this paper concludes the deficiencies of traditional management model, analyzes requirements of lab and describes the design of management system. It lays a good foundation for implementing the intelligent management of electrical and electronic laboratory.
\end{abstract}

\section{Introduction}

Due to large amounts of laboratory materials and business, the prior method of manual management is unable to achieve the expected query and management efficiency. Except teaching and researching work, the instruments and equipments remain idle. And this causes a waste of devices. Because of the large quantity and various types of the equipments, it is very difficult to do some statistical analysis. The traditional management methods of electrical and electronic laboratory are relatively closed, and the resources have not been shared by the teachers and students. It leads to problems that the equipments and sites of laboratory cannot meet the needs of teaching and research. An openly integrated query system for devices and consumables has not been established, and the statistics of material consumption cannot be compiled in detail. The laboratory security management is only sustained by artificial supervision, which is unable to satisfy the requirement of modernized comprehensive laboratory. In addition, the students' safe consciousness is weak, which increases the number of potential safety problems [1-2].

On the other hand, the laboratory regards the operational and verified experiments as principle and the comprehensive and designing experiments are free from value. It leads to lack effective communication between teachers and students. Meanwhile, it makes students lose interest in doing experiments, refraining students from displaying their originality and creativity. The lab managers are influenced by the concept that the professional knowledge is more important than basic that, the theory is more significant than practice, which results in their low work motivation [3-4].

For application-oriented university, it demands high quality education and the efficiency, scale and form of laboratory management will be faced with fierce tests. Therefore, the scientific and modern management of laboratory is essential trend.

\section{Function of Management System}

Above disadvantages of the traditional management pattern should be improved. The managers should be released from the heavy management workload, and have more time to engage in the development of new experimental project and scientific research. Simultaneously, in order to train applied talents who have abilities of analyzing and solving problems independently, the following system functions are determined. The functional structure of the system is shown in Figure1.

A. Equipment management system

The model, parameters, principle diagrams, and instructions of equipments are loaded into database for upgrading and repairing. For new and existing equipments, their overall lifecycle 
profiles are built. And the custodians, usages, and maintenances of equipments are noted in their files. The their status is updated in real time, such as used, not used, outside, or idle. In the meantime, the frequent problems and solutions of equipments are recorded to get information for purchasing components. In this, the time of device maintenance is reduced when the equipment has faults and the equipment utilization is enhanced.

The electrical and electronic laboratory involves a large number of consumable goods, such as electric tools, wires, components, solders, rosins, etc. The name, type, quantity, and location of low-value consumables are imported into the system database. And their information should be real-time update to make teachers and students look up the required materials online conveniently, which keeps the experiment working properly. And the information of claiming goods will be recorded in the user account, which makes people enhance accountability. Through this method, the issues such as the repeat purchase, shortage of consumables in types, waste caused by idle for a long time are resolved.

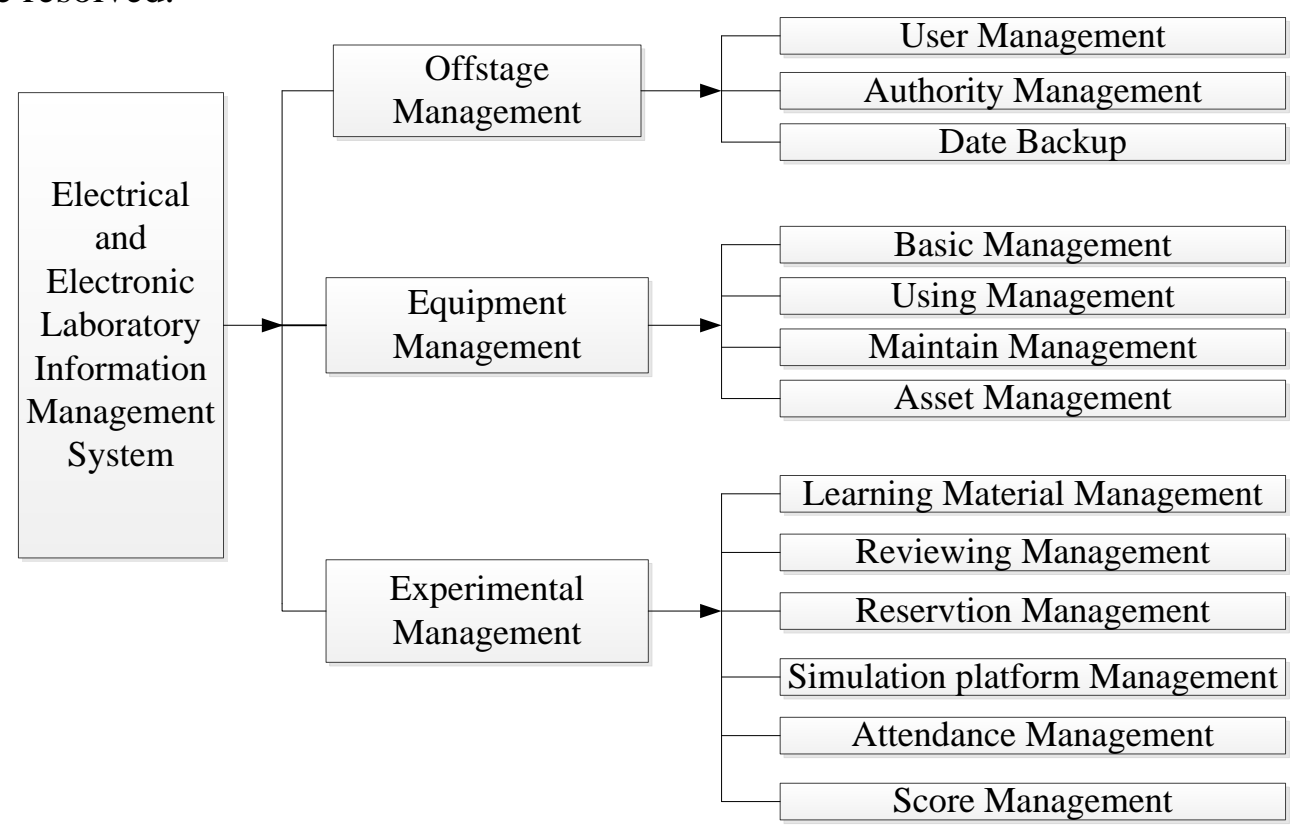

Fig.1. The functional structure of system

B. Booking system of experiments on the network

The experimental content is divided into two parts: one is basic experiments, the other is extended experiments. The students can select the experiments according to their requirements on the network. And in general, the students do not preview the experiment contents before doing it. So, the results of experiment are not necessarily satisfying. In this management system, the students cannot reserve the experiment until pass the examination. The strategy enhances the experimental efficiency, and reduces the damage of equipments and instruments as well as.

C. Online learning system

The modern long-distance open education is used to improve experimental teaching effect. Through the combination of computer, mobile client and network, the educational videos are posted on the web. Because of its rich images, texts and voices, the more spirited effective education form is provided to help students for learning, memory and understanding. Besides, the final experiment report can be submitted and the scores and remarks can be queried online [5].

D. Online simulation platform

The platform can simulate the equipments and facilities that are used in the actual experiment, and offer the experimental environment which is similar to the real experiment. The advantages are less investment and low maintenance, and it is not limited by the conditions including time, location, human and material resources. Some design-based experiments can be realized by simulation, firstly. Then, the real platform is built in the laboratory.

E. Authentication

The way that combines smart card with image acquisition authentication is adopted, and it 
realizes attendance management and identifying the students, which prevents false presence. In addition, the general power supply of the instrument is turned on through the card that is licensed.

\section{System Performance Index}

A. The system should be stable and reliable.

A single server should support more than 10000 registered users and 500 concurrent users. If the system fails, the teaching process and schedule will be affected. Therefore, the system must be stably running.

B. The system should have the characteristic of sustainable development.

With rapid development of the laboratory, the management system should update and upgrade constantly. It should have the optimizing function to develop continually [6].

C. The system should have high security and well dependent.

The safety and efficient authentication and authority checking can prevent from the leaks of system data and the outsider illegal intrusion. At the same time, the system data should be backed up to ensure the system normally runs in case system trouble occurs [7].

D. The system should be easy to operate and maintenance.

The system should have friendship of interfaces, simply operate, conveniently etc.

\section{Topological Structure of System}

According to the function of management system, the topological structure of electrical and electronic laboratory is divided into three layers: perceptual layer, network layer, and application layer. The topological structure of system is shown in Fig2.

The perceptual layer is composed of many sensor networks, or the sentient MZM (Machine to Machine) that can be the communication terminal with perception ability or the sensors with reading and writing ID card. The related techniques of perceptual layer in this system are RFID (Radio Frequency Identification) and image acquisition technology. The former is a kind of communication technology, that the mechanical or optical contact between specific targets is established through radio signals. The principle of the later is that the optical image taken by the watching cameras is converted to video signal, which is digitalized by the image acquisition card. Then the digital image data is generated, that is processed and preserved by computer.

The network layer is constituted by private network, internet, wired and wired network, network management system, cloud computing platform, etc. It accomplishes the transmission, routing and control of information mainly, that includes connect and core network. After the infrastructures and products are perceived in perceptual layer, the network layer will be responsible for transmitting and processing the information.

The application layer that is combined with industry demands is the interface between systems and users. And it can realize the intelligence application of system [8].

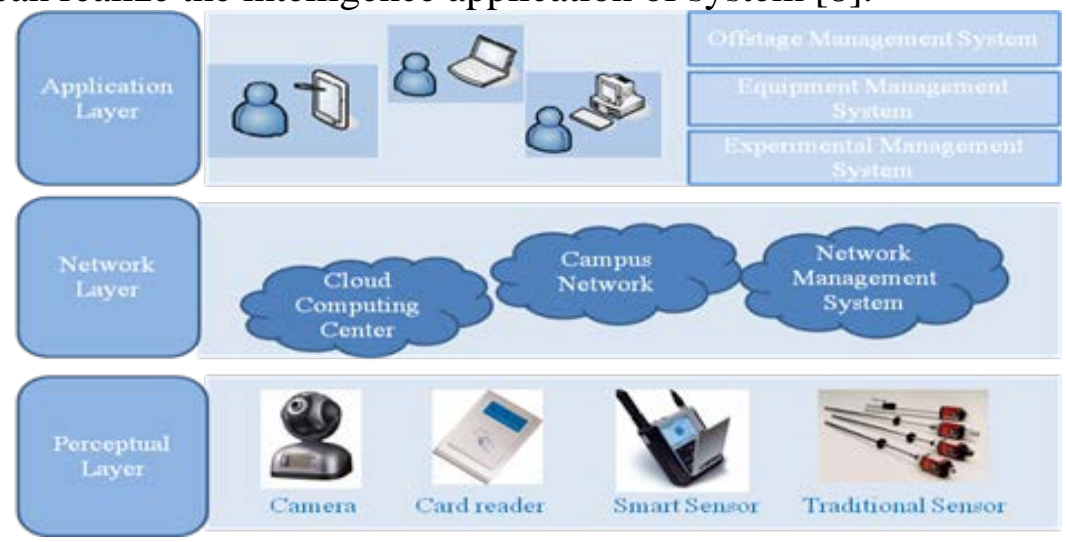

Fig.2. The topological structure of system 


\section{Conclusion}

This paper designs the electrical and electronic laboratory management system for application-oriented university preliminarily. The lab manager can manage the equipments and instruments visa the management platform, which reduces duplicate work. The students can learn online, make an appointment, simulate, submit the report, and search grade of experiment. Besides, it has the characteristics of modern management, and meets the requirements of the laboratory management. The problem about the insufficient resource of teaching is solved, the students' own initiatives are activated, and the laboratory teachers' vocational level is raised. In conclusion, this system has many advantages, and is worth of popularizing and applying.

\section{Acknowledgement}

In this paper, the research was sponsored by the Seed Foundation of Shanghai University of Medicine and Health Sciences (Project No. HMSF-16-12-012).

\section{References}

[1] Liu Xia. Application of RFID Technology in the Open Electrical and Electronic Laboratory Management System[J]. Experiment Science and Technology, 2014, 12(6)52-56.

[2] Shi Shuyou, Bai Yang, Jiang Chunxia, Li Minghua. Research on Teaching and Management System for Electrical and electronic Experiments [J]. Experimental Technology and Management, 2008, 25(10)81-43.

[3] Qu Wei, Qiu Chengjun, Liu Mingliang, Yunxia Wang. Construction and Practice of the Practical Teaching System of Electrical and Electronic Experimental Teaching Center[J]. Research and Exploration in Laboratory, 2010, 29 (7) 216-224.

[4] He Zhenlei, Zhu Shan'an.Design and Implementation of An Internet-based Electrical Engineering Laboratory[C]. IEEE International Conference on Control and Automation.HangZhou:China,2013,176-179.

[5] Zhang Huan, Zhu Shanan. Internet-based Electrical Engineering Lab Integrates Real and Virtual Experiments[C].The $8^{\text {th }}$ International Conference on Computer Science\&Education.Colombo:Sri Lanka, 2013,11-15.

[6] Cai Mingshan. Researches and Designs on Collegial Laboratory Management System [D].Hunan: Hunan University, 2003.

[7] Jia Hongjian. The Design and Implementation of Management Information System for Labor Security Supervision[D]. Chengdu: University of Electronic Science and Technology of China, 2014.

[8] Zhong Xinmei. Integrated Management System Design of Electrical and Electronic Information Experiment Education Center[D]. Chengdu: Southwest Petroleum University,2014. 